\title{
Nonlocal thermoelectricity in a Cooper-pair splitter
}

\author{
Robert Hussein, ${ }^{1}$ Michele Governale, ${ }^{2}$ Sigmund Kohler, ${ }^{3}$ Wolfgang Belzig, ${ }^{1}$ Francesco Giazotto, ${ }^{4}$ and Alessandro Braggio ${ }^{4}$ \\ ${ }^{1}$ Fachbereich Physik, Universität Konstanz, D-78457 Konstanz, Germany \\ ${ }^{2}$ School of Chemical and Physical Sciences and MacDiarmid Institute for Advanced Materials and Nanotechnology, Victoria University \\ of Wellington, P.O. Box 600, Wellington 6140, New Zealand \\ ${ }^{3}$ Instituto de Ciencia de Materiales de Madrid, CSIC, Cantoblanco, E-28049 Madrid, Spain \\ ${ }^{4}$ NEST, Istituto Nanoscienze-CNR, Piazza S. Silvestro 12, Pisa I-56127, Italy
}

(Received 12 June 2018; published 19 February 2019)

\begin{abstract}
We investigate the nonlocal thermoelectric transport in a Cooper-pair splitter based on a double-quantum-dotsuperconductor three-terminal hybrid structure. We find that the nonlocal coupling between the superconductor and the quantum dots gives rise to nonlocal thermoelectric effects which originate from the nonlocal particle-hole breaking of the system. We show that Cooper-pair splitting induces the generation of a thermocurrent in the superconducting lead without any transfer of charge between the two normal-metal leads. Conversely, we show that a nonlocal heat exchange between the normal leads is mediated by nonlocal Andreev reflection. We discuss the influence of finite Coulomb interaction and study under which conditions nonlocal power generation becomes possible, and when the Cooper-pair splitter can be employed as a cooling device.
\end{abstract}

DOI: 10.1103/PhysRevB.99.075429

\section{INTRODUCTION}

Hybrid superconductor devices [1-16] are promising candidates for entanglement generation in solid-state systems and, therefore, have potential applications for superconducting spintronics [17], quantum information, and quantum computation $[18,19]$. The central idea is that the electrons in an $s$-wave superconductor are in a spin-entangled state which can be made electronically accessible by splitting them via cross-Andreev reflection (CAR) into spatially separated normal leads. The competing process of local Andreev reflection (LAR), where the electrons tunnel into the same lead, does not directly contribute to the spatially nonlocal entanglement. In order to increase the CAR fraction of the current and minimize the effect of LAR, different strategies have been adopted such as employing ferromagnetic leads [20-24] or including quantum dots with large intradot Coulomb repulsion [25-32]. In double quantum dots with finite Coulomb repulsion, the possibility has been discussed of inducing spatially nonlocal entanglement and manipulating its symmetry by involving only the LAR process even without the nonlocal coupling $[33,34]$.

The study of energy harvesting has also drawn much attention over the last few years [35-39]. Among the suggested implementations using superconductors are S-N junctions [40], ferromagnet hybrid systems [41-46], and hybrid quantum-dot systems [47-50]. Aspects such as thermodynamic efficiencies [51-59] and thermoelectric effects in strongly correlated quantum dots $[60,61]$ have been addressed. In particular, Machon et al. suggested in Ref. [41] that nonlocal thermoelectric effects in Cooper-pair splitters should exist. Furthermore, Cao et al. showed in Ref. [62] that Cooper-pair splitting can be achieved in the absence of bias voltages by applying a thermal gradient to the normal leads. Inspired by this idea, we present in this work a detailed study of the nonlocal thermoelectric properties of a Cooper-pair splitter taking fully into account the Coulomb interaction. Further, we discuss the possibility of nonlocal cooling and power generation. Intriguingly, we show that the system still becomes a thermoelectric device due to the influence of the superconducting lead, which by itself is not thermoelectrically active being intrinsically particle-hole symmetric. This is essentially due to the fact that the nonlocal particle-hole symmetry is broken as a consequence of the thermal gradient and the three-terminal device geometry.

This work is organized as follows. In Sec. II, we introduce our model and the formalism employed to calculate the thermoelectric properties. We explore the thermoelectric properties in the linear regime in Sec. III, and compare the results to simplified effective models. Section IV is devoted to the study of nonlocal power generation and cooling. Finally, we draw our conclusions in Sec. V.

\section{MODEL AND MASTER EQUATION}

In this section we introduce the model of the Cooper-pair splitter, sketched in Fig. 1, and the formalism employed to calculate its thermoelectric properties. The Cooper-pair splitter is composed of two quantum dots coupled to an $s$-wave superconductor and two normal-metal leads; see Ref. [33]. For a large superconducting gap, $|\Delta| \rightarrow \infty$, the subgap physics is described by the effective Hamiltonian [33,63-69]

$$
\begin{aligned}
H_{S}= & H_{\mathrm{DQD}}-\sum_{\alpha=L, R} \frac{\Gamma_{S \alpha}}{2}\left(d_{\alpha \uparrow}^{\dagger} d_{\alpha \downarrow}^{\dagger}+\text { H.c. }\right) \\
& -\frac{\Gamma_{S}}{2}\left(d_{R \uparrow}^{\dagger} d_{L \downarrow}^{\dagger}-d_{R \downarrow}^{\dagger} d_{L \uparrow}^{\dagger}+\text { H.c. }\right),
\end{aligned}
$$

where $H_{\mathrm{DQD}}$ describes the double-quantum-dot (DQD) system; the second term characterizes the local Cooper-pair tunneling between the superconductor and $\operatorname{dot} \alpha=L, R$ with 


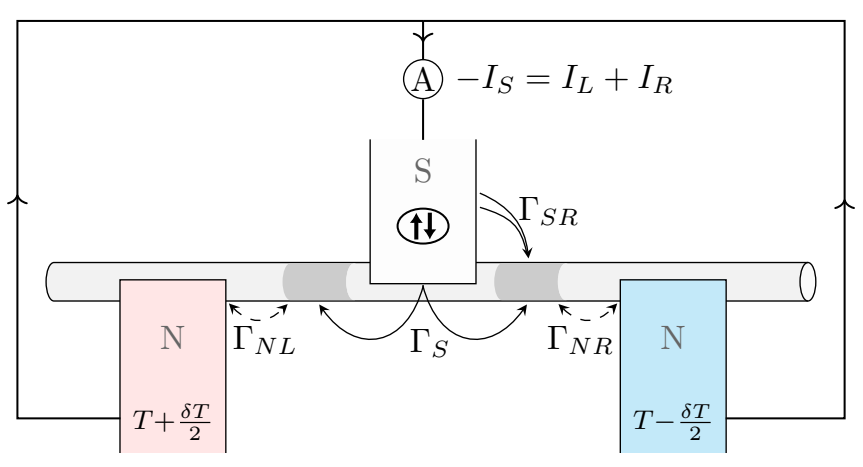

FIG. 1. Cooper-pair splitter circuit consisting of a double quantum dot coupled to two normal leads $(\mathrm{N})$ and a superconducting one (S). A temperature gradient $\delta T$ between the normal leads induces a nonlocal current into the superconductor which may generate power for finite chemical potentials.

tunneling rates $\Gamma_{S \alpha}$. Here, $d_{\alpha \sigma}^{\dagger}$ denotes the fermionic creation operator for an electron on dot $\alpha$ with spin $\sigma=\uparrow, \downarrow$. The last term describes the nonlocal tunneling of a Cooper-pair splitting into both dots with the rate $\Gamma_{S} \sim \sqrt{\Gamma_{S L} \Gamma_{S R}} e^{-l / \xi}$. The nonlocal coupling $\Gamma_{S}$ becomes large when the distance $l$ between both quantum dots is small compared to the coherence length $\xi$ of the superconductor. The DQD is modeled by

$$
H_{\mathrm{DQD}}=\sum_{\alpha, \sigma} \epsilon_{\alpha} n_{\alpha \sigma}+\sum_{\alpha} U_{\alpha} n_{\alpha \uparrow} n_{\alpha \downarrow}+U \sum_{\sigma, \sigma^{\prime}} n_{L \sigma} n_{R \sigma^{\prime}}
$$

with $\epsilon_{\alpha}$ the orbital energies and $U_{\alpha}(U)$ the intradot (interdot) Coulomb interaction; $n_{\alpha \sigma}=d_{\alpha \sigma}^{\dagger} d_{\alpha \sigma}$ is the occupation operator. We note that in the limit $|\Delta| \rightarrow \infty$, the system Hamiltonian is exact in the superconducting coupling $\Gamma_{S}$ [64]. This model assumes large single-level spacings in the quantum dots. Hence, a maximum of two electrons with opposite spin can occupy each dot, and in total at most four electrons can reside in the DQD. In the following, we mainly focus on the nonlocal resonance which is not substantially affected by the Coulomb interaction when $U \ll U_{\alpha}$. In this regime the nonlocal resonance occurs for gate voltages $\epsilon_{\alpha} \approx-U / 2$, as discussed in Ref. [33]. Hereafter, in general we consider the case of $U=0$, but the generalization to finite interdot Coulomb interaction $U \neq 0$, however, is straightforward.

\section{Master equation and transport coefficients}

For the computation of particle and heat currents, we restrict ourselves to the sequential tunneling regime, $\Gamma_{N L}, \Gamma_{N R} \ll k_{B} T_{\alpha}$, with $T_{\alpha}$ being the temperature of the normal lead $\alpha$. Moreover, we consider the case of weak coupling to the normal leads, $\Gamma_{N \alpha} \ll \Gamma_{S}$, and thus can express the populations $P_{a}$ of the eigenstates $|a\rangle$ of the system $\left(H_{S}|a\rangle=\right.$ $\left.E_{a}|a\rangle\right)$ by a Pauli-type master equation of the form [33] $\dot{P}_{a}=\sum_{a^{\prime}}\left(w_{a \leftarrow a^{\prime}} P_{a^{\prime}}-w_{a^{\prime} \leftarrow a} P_{a}\right)$ with the stationary solution denoted as $P_{a}^{\text {stat }}$. With increasing $\Gamma_{N \alpha}$, one may have to consider off-diagonal density matrix elements [70,71] or work in a local basis $[72,73]$. The transition rates for tunneling of an electron from the normal lead $\alpha$ to the respective $\operatorname{dot}(s=+1)$ and the opposite processes $(s=-1)$ are simply given by
Fermi's golden rule

$$
w_{a \leftarrow a^{\prime}}^{(\alpha, s)}=\sum_{\sigma} \Gamma_{N \alpha} f_{\alpha}^{(-s)}\left(-s \omega_{a a^{\prime}}\right)\left|\left\langle a\left|d_{\alpha \sigma}^{(-s)}\right| a^{\prime}\right\rangle\right|^{2},
$$

with the notation $d_{\alpha \sigma}^{(-s)}$ for the electron creation and annihilation operators, $\omega_{a a^{\prime}}=E_{a}-E_{a^{\prime}}$, and $f_{\alpha}^{(s)}(\epsilon)=\{1+\exp [s(\epsilon-$ $\left.\left.\left.\mu_{\alpha}\right) / k_{B} T_{\alpha}\right]\right\}^{-1}$ for the Fermi function at the chemical potential $\mu_{\alpha}$. Hereafter, we fix the chemical potential of the superconductor to be zero, $\mu_{S C}=0$, using it as reference for the chemical potentials of the normal leads, $\mu_{\alpha}$. Hence, the total rates entering the master equation are given by

$$
w_{a \leftarrow a^{\prime}}=\sum_{\alpha, s= \pm} w_{a \leftarrow a^{\prime}}^{(\alpha, s)}
$$

The electron and heat currents through the contacts correspond to the rates of changes of the particle number and the energy in the corresponding lead, $I_{\alpha} \equiv e_{0}\left\langle\dot{N}_{\alpha}\right\rangle$ and $\dot{Q}_{\alpha}$, respectively. In the sequential-tunneling regime with the normalmetal leads it is easy to write the currents in terms of the stationary populations of the DQD $P_{a^{\prime}}^{\text {stat }}$ and the rates $w_{a \leftarrow a^{\prime}}^{(\alpha, s)}$ :

$$
\begin{gathered}
I_{\alpha}=\frac{e_{0}}{\hbar} \sum_{a, a^{\prime}, s= \pm} s w_{a \leftarrow a^{\prime}}^{(\alpha, s)} P_{a^{\prime}}^{\mathrm{stat}}, \\
\dot{Q}_{\alpha}=-\frac{1}{\hbar} \sum_{a, a^{\prime}, s= \pm}\left(E_{a}-E_{a^{\prime}}\right) w_{a \leftarrow a^{\prime}}^{(\alpha, s)} P_{a^{\prime}}^{\mathrm{stat}}-\frac{\mu_{\alpha}}{e_{0}} I_{\alpha} .
\end{gathered}
$$

The last term in Eq. (6) reflects the fact that, in order to obtain the heat current, one needs to subtract the net energy associated with the flux of particles at the fixed electrochemical potential $\mu_{\alpha}$. For the superconducting leads, the electric current is determined by current conservation, that is, $I_{S}=$ $-I_{L}-I_{R}$. In the large-gap limit, due to perfect Andreev heat mirroring, the heat transferred to the superconductor vanishes, i.e., $\dot{Q}_{S}=0$. This means that the heat current in the system flows only between the normal leads.

In thermoelectrical systems, it is instructive to discuss the linear regime at small voltages and small thermal biases. Thus, the linear response of the electric currents and the heat currents of our three-terminal system can be described by six equations, which reduce to four equations, when taking into account particle and energy conservation $[55,58,74]$. Since for a large superconducting gap heat can be exchanged only between the normal leads but no electric current can flow between them at the nonlocal resonance at equal chemical potentials, we will restrict ourself to the following relations:

$$
\begin{gathered}
\delta I_{S}=L_{11}^{S} \delta V+L_{12}^{S} \delta T, \\
\delta \dot{Q}_{R}=L_{21}^{R} \delta V+L_{22}^{R} \delta T,
\end{gathered}
$$

with four transport coefficients $L_{i j}^{S / R}, \delta V=\left(\mu_{L}+\mu_{R}\right) / 2 e_{0}$, and $\delta T=T_{L}-T_{R}$ [75]. Here, $-\delta V /\left.\delta T\right|_{I_{\alpha}=0}=L_{12}^{S} / L_{11}^{S}$ defines the nonlocal Seebeck coefficient, which, multiplied by the temperature difference $\delta T$, yields the nonlocal Seebeck potential, and $\delta \dot{Q}_{R} /\left.\delta T\right|_{\mu_{\alpha}=0}=L_{22}^{R}$ is the closed circuit thermal conductance of the right normal lead. For a multiterminal generalization, see Refs. [55,58]. Beyond the linear regime, Onsager coefficients of higher order $[76,77] L_{k k^{\prime}}^{S}, L_{k k^{\prime}}^{R}$ can be calculated by recursive methods [78]. The masterequation formalism presented so far can be easily gener- 
alized to compute higher-order current cumulants by using standard full-counting-statistics techniques and introducing appropriate counting variables both for the charge and energy currents [78-84]. Hereafter, we only consider the average currents since these are the quantities that are easily accessible experimentally.

\section{NONLOCAL THERMOELECTRICITY}

Hereafter, we will discuss the nonlocal thermoelectrical behavior of the Cooper-pair splitter for intradot Coulomb energies $U_{R}$ and $U_{L}$ much larger than any other energy scale, such that double occupancy of each individual dot is energetically forbidden. This simplifies the system making the physics more transparent [85]. In order to investigate thermoelectrical effects, we assume that the normal leads are at different temperatures, $T_{L}>T_{R}$. We focus on the following nonlocal thermoelectrical effect: A thermal gradient between the normal leads induces a charge current between the superconductor and the normal leads, see Fig. 1, even if the chemical potentials of the three leads are kept equal, $\mu_{S C}=\mu_{R}=\mu_{L}=0$. In the limit $U_{R}, U_{L} \gg \epsilon_{R}, \epsilon_{L} \approx 0$, the current through the superconducting lead $I_{S}$ is purely induced by nonlocal Cooperpair splitting, $I_{S}<0$, and recombination, $I_{S}>0$, respectively. In the former (latter) process Cooper pairs, consisting of electron singlets, split into (recombine from) different dots. Since only nonlocal Andreev reflection is present, the average currents through the two normal leads are identical, $I_{R}=$ $I_{L}=-I_{S} / 2$, irrespectively of the lead temperatures and tunnel couplings.

Furthermore, since we consider the situation of a large superconducting gap, $|\Delta| \rightarrow \infty$, no quasiparticle excitation can take place and heat transfer within the superconducting lead is forbidden. Thus, heat transfer can only occur between the two normal leads mediated by the superconducting lead, which operates as a perfect nonlocal Andreev mirror.

In Fig. 2(a), we show a density plot of the superconducting current $I_{S}$ as a function of the level energies $\epsilon_{R}$ and $\epsilon_{L}$ for temperatures much smaller than the nonlocal coupling, $k_{B} T_{\alpha} \ll \Gamma_{S}$. We recognize immediately that the current is finite for $\epsilon_{R} \neq \epsilon_{L}$. The current is nonvanishing close to the dashed lines corresponding to the resonance conditions $2 \Delta E_{ \pm}=\epsilon_{L}-\epsilon_{R} \pm \sqrt{\left(\epsilon_{L}+\epsilon_{R}\right)^{2}+2 \Gamma_{S}^{2}}=0$.

The addition energies, $\Delta E_{ \pm}$, correspond to processes of electron exchange at the normal leads for the model Hamiltonian

$$
H_{\mathrm{eff}}=\sum_{\alpha \sigma} \epsilon_{\alpha}\left(|\alpha \sigma\rangle\langle\alpha \sigma|+\frac{|S\rangle\langle S|}{2}\right)-\frac{\Gamma_{S}}{\sqrt{2}}(|0\rangle\langle S|+| S\rangle\langle 0|)
$$

following from Eq. (1) when restricting it to the subspace involving the empty state $|0\rangle$, the singly occupied states $|\alpha \sigma\rangle=$ $d_{\alpha \sigma}^{\dagger}|0\rangle$ of dot $\alpha=L, R$ with spin $\sigma=\uparrow, \downarrow$, and the singlet state $|S\rangle=\frac{1}{\sqrt{2}}\left(d_{R \uparrow}^{\dagger} d_{L \downarrow}^{\dagger}-d_{R \downarrow}^{\dagger} d_{L \uparrow}^{\dagger}\right)|0\rangle$. In the Hamiltonian we have omitted the triplet states as they cannot be directly coupled to the superconductor, where only singlet Cooper pairs are present. The triplet states play an important role in the high-bias regime, yielding a suppression of the current called triplet blockade [65]. They are also crucial in the presence of interdot tunneling in combination with spin-orbit interaction [33,34].

In Fig. 2(b), we consider the heat flow from the hot to the cold normal lead. Essentially, nonvanishing heat flow occurs where the thermoelectric behavior is present, indicating that the mechanism of thermoelectricity in the system is also responsible for the heat exchange. Intriguingly, here the heat exchange is mediated only by Cooper pairs, since there is no other way for an excitation to be transferred from a normal lead to another normal lead without a process involving a Cooper-pair emission or absorption at the superconducting interface. As expected, Cooper pairs cannot transfer heat to/from the superconductor, being at zero energy (ground state), but they can coherently mediate heat exchange between the normal leads. We refer to this mechanism as nonlocal heat exchange coherently mediated by the superconducting lead. This interpretation is supported by the fact that the heat exchanged is enhanced just inside the gap between the two resonances; see Fig. 2(b). Indeed, inside the gap for $\epsilon_{R}$, $\epsilon_{L} \approx 0$ the heat current remains finite. This is a consequence of the fact that the contributions of the two nearby resonances have opposite particle/hole character and add up. Conversely, the thermoelectrical current is suppressed in the gap since particles and holes have opposite charges and, consequently, yield opposite contributions to the thermoelectrical current. It is important to stress that this heat transfer mediated by the superconductor does not affect the superconducting state and can be interpreted as a nonlocal version of the Andreev mirror phenomena for the heat current.

In panels (c) and (d) of Fig. 2, we consider the linear regime in the temperature, $\delta T \ll T=\left(T_{L}+T_{R}\right) / 2$. In this case, the linear transport coefficients depend only on the average temperature $T$ of the leads. We now discuss how the linear coefficients $L_{12}$ and $L_{22}$ vary with the detuning $\Delta \epsilon \equiv \epsilon_{R}-\epsilon_{L}$ along the line $\epsilon_{L}=-\epsilon_{R}$ when changing the temperature but keeping fixed the nonlocal coupling $\Gamma_{S}$, which determines the distance between the two resonances.

In Fig. 2(c) the two central (inner) peaks progressively cancel each other as the temperature increases. This is a consequence of the fact that when $\Gamma_{S} \gtrsim k_{B} T$, the electronlike contribution of a resonance coexists with the hole-like contribution of the other resonance. This competition reduces the total thermoelectric current. Once $k_{B} T=\Gamma_{S}$ the two resonances merge and behave as a single resonance. Panel (d) shows the corresponding linearized heat current coefficient $L_{22}^{R}$. For well-separated peaks, $\Gamma_{S} \gg k_{B} T$, the heat current essentially vanishes at the resonances, where also the current coefficient vanishes. In the situation when the peaks are in proximity, $\Gamma_{S} \gtrsim k_{B} T$, they add up constructively at $\Delta \epsilon \approx 0$. When $k_{B} T=\Gamma_{S}$ again the thermal behavior resembles the contribution of a single QD resonance.

Let us develop a physical picture to explain the behavior of the linear thermoelectric coefficients in the limit of large intradot Coulomb interaction, for equal chemical potentials, $\mu \equiv \mu_{L}=\mu_{R}$, and in the presence of a temperature gradient between the two normal leads, $T_{L}>T_{R}$. Figure 3(a) depicts the level structure of the double-quantum-dot system in the situation where the chemical potentials of the normal leads coincide with the one of the superconductor. For $\epsilon_{L}, \epsilon_{R} \approx 0$ the singlet state mixes with the empty state forming nonlocal 

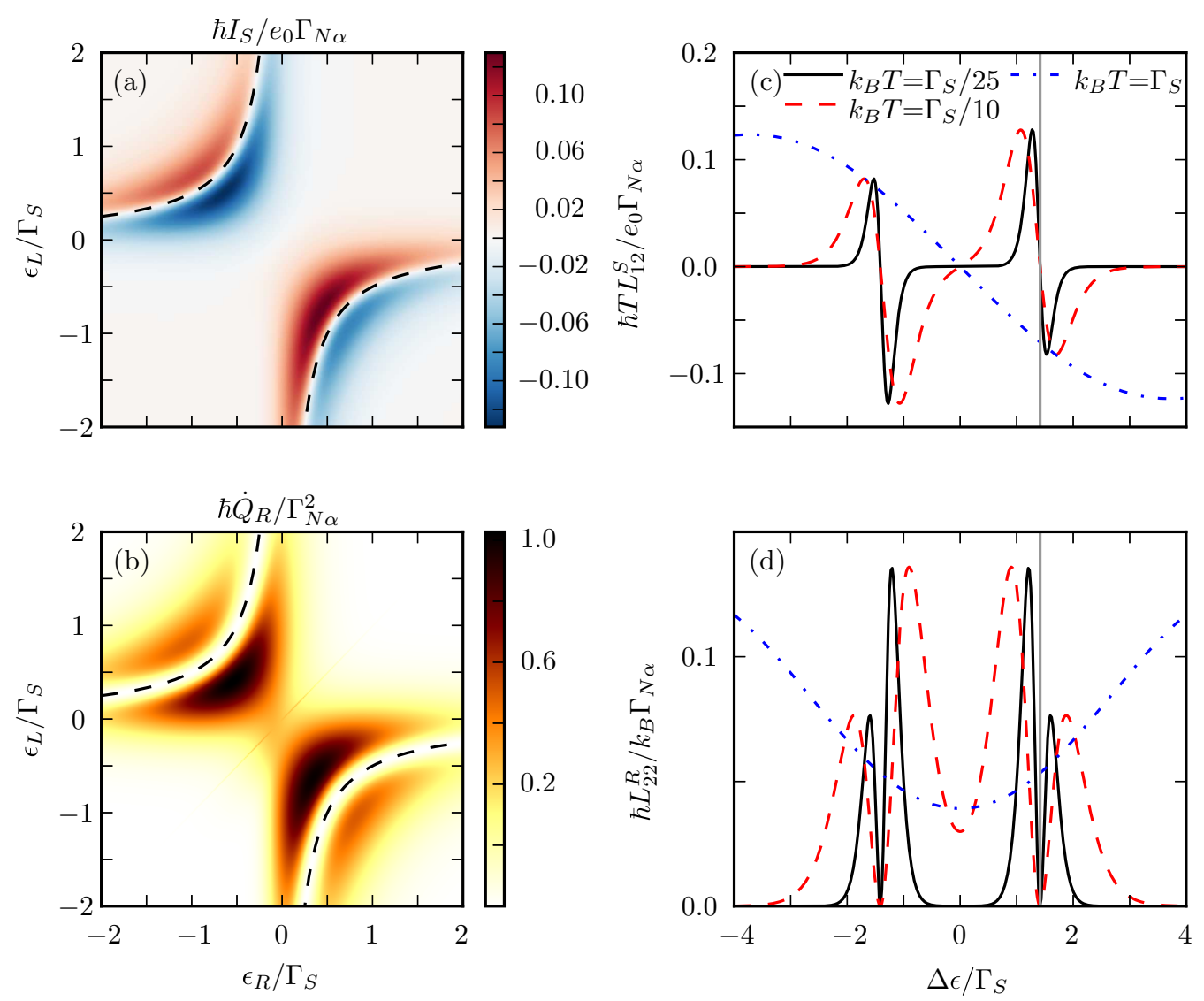

FIG. 2. (a) Superconducting current $I_{S}=-I_{L}-I_{R}$ and (b) heat current $\dot{Q}_{R}$ through the right normal lead as a function of the level energies $\epsilon_{L}$ and $\epsilon_{R}$. Parameters are $k_{B} T_{L}=15 \Gamma_{N \alpha}, k_{B} T_{R}=5 \Gamma_{N \alpha}, \Gamma_{S}=100 \Gamma_{N \alpha}, U=\mu_{\alpha}=0, \Gamma_{S \alpha}=\Gamma_{S}$, and $U_{\alpha} \gg \Gamma_{S}$. The dashed lines indicate where the Andreev bound state addition energies $\left[\left(\epsilon_{L}-\epsilon_{R}\right) \pm \sqrt{2 \Gamma_{S}^{2}+\left(\epsilon_{L}+\epsilon_{R}\right)^{2}}\right] / 2$ are resonant with the Fermi levels at $\mu=0$. (c), (d) Linear current coefficient $L_{12}^{S}$ and heat current coefficient $L_{22}^{R}$ in the limit $\delta T \equiv T_{L}-T_{R} \rightarrow 0$ as a function of the detuning $\Delta \epsilon \equiv \epsilon_{R}-\epsilon_{L}$ for different average temperatures $T$. Here, the energy levels are symmetrically detuned, i.e., $\epsilon_{R}=-\epsilon_{L}$. The vertical line indicates one of the Andreev bound state addition energies.

Andreev bound states shared between the two dots due to nonlocal Cooper-pair tunneling with the central superconducting lead [86]. The electron tunneling with the normal leads determines transition between the DQD states. The dotted lines indicate the Andreev bound state addition energies $\Delta E_{ \pm}$, while the solid lines indicate the orbital energy levels of the quantum dots.

For the case under consideration $\left(T_{L}>T_{R}\right)$, when more electrons are above the Fermi level of the left normal lead than holes below the Fermi level of the right normal lead, electrons tunnel via the Andreev bound-state channel into the superconductor and form Cooper pairs. At the same time Cooper pairs can split in an opposite process and tunnel into the normal leads. The difference of both processes yields a net thermoelectrical current when the normal leads have different temperatures. This effect is a direct consequence of the nonlocal particle-hole asymmetry induced by the structure.

\section{A. Linear regime and effective models}

In Fig. 4, we show in solid lines the dependence of the linear coefficients on the nonlocal coupling $\Gamma_{\mathrm{S}}$ keeping fixed the temperature $T$. This behavior can be roughly interpreted by mapping the Cooper-pair splitter in the CAR regime to a simplified model of a single quantum dot with two resonances located at the addition energies of the Andreev bound states; see the inset of Fig. 4. We observe that the thermoelectric behavior of this simple model resembles the thermoelectric behavior of the full one. Its main aim is to show that the linear behavior of the full system is not too different from the conventional thermoelectrical properties of a quantum dot system, even though the former is mainly characterized by the discussed nonlocality in the heat and charge transport which cannot be present in a simple quantum dot. The thermoelectrical coefficients for the simplified model can be expressed in the Landauer-Büttiker formalism as [87-91]

$$
L_{k+1,2}^{\mathrm{eff}} \equiv \frac{\left(2 e_{0}\right)^{1-k}}{h} \int_{-\infty}^{\infty} d E \frac{E^{k+1} \tau(E)}{4 k_{B} T^{2} \cosh ^{2}\left(E / 2 k_{B} T\right)} .
$$

The additional factor 2 for the electron current $(k=0)$ takes into account that in an Andreev process the current is doubled $\left(I_{S}=-2 I_{R}\right)$. The transmission function is modeled by two Lorentzians located at the Andreev bound state energies

$$
\tau(E) \propto \sum_{s= \pm} \frac{\gamma}{\left(E-\Delta E_{s}\right)^{2}+(\gamma / 2)^{2}} .
$$




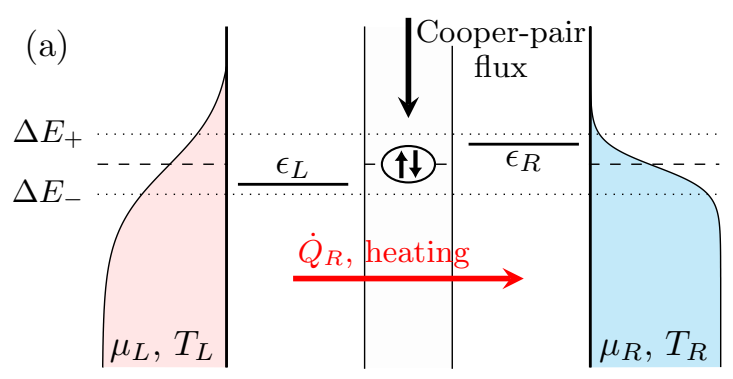

(b)

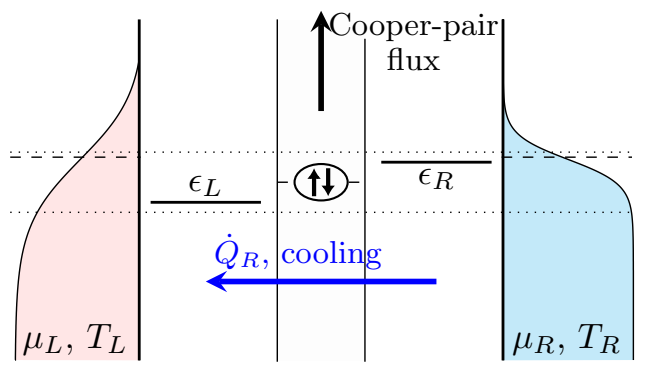

FIG. 3. (a) Cooper-pair splitter configuration for zero chemical potentials, $\mu_{L}=\mu_{R}=0$, leading to a heating of the right normal lead (red arrow) and a net flux of Cooper pairs from the superconductor into the normal leads (black arrow). (b) Configuration for finite chemical potentials, $\mu_{L}=\mu_{R}>0$. A net flux of Cooper pairs flows into the superconductor (black arrow) against the intrinsic thermocurrent leading to a cooling of the right normal lead (blue arrow).

Finally we arrive at $L_{k+1,2}^{\text {eff }}=\frac{\alpha}{\hbar}\left(2 e_{0}\right)^{1-k} \sum_{s= \pm} A_{k}\left(\Delta E_{s}\right)$ with $\alpha$ an overall scaling factor and for $k=0, \pm 1$ the function

$$
A_{k}(\Omega)=\sum_{s= \pm} \frac{\omega_{s}^{k+1}(\Omega)}{k_{B}(2 \pi T)^{2}} \Psi^{\prime}\left(\frac{1}{2}-\frac{s i \omega_{s}(\Omega)}{2 \pi k_{B} T}\right)+\frac{\gamma \delta_{k, 1}}{2 \pi T},
$$

which collects the contributions from the poles of the Fermi function and the poles $\omega_{s}(\Omega)=\Omega+s i \gamma / 2$ of the Lorentzians. Here, $\Psi$ denotes the digamma function.

The reader should be aware that in the quantum-dot model the thermoelectrical current and the thermal current always flow between the two normal leads. In the full system, instead, due to the presence of the superconducting lead with a nonlocal coupling, the charge and the thermal current flow in different terminals enabling, thus, nonlocal thermoelectricity [74].

In Fig. 4, we compare the linear thermoelectric coefficient [panel (a)] and the thermal conductance [panel (b)] of this simplified model (dashed lines) with the results of the full calculation (solid lines) for different nonlocal couplings $\Gamma_{S}$ as a function of the detuning. Here, we fix the free parameter $\alpha$ of the mapped quantum-dot model such that the linear current coefficient at $\Gamma_{S}=k_{B} T$ fits the one of the full model. The mapped quantum-dot model qualitatively captures the curve progression of the full computation. When the nonlocal coupling is much larger than the temperature (blue lines) the behavior exhibits two well-separated resonances. At $\Gamma_{S}=$ $4 k_{B} T$ (green lines), the heat transport around zero detuning is enhanced and this can be understood by the additive superposition of the contributions of both Lorentzians. For lower values of the nonlocal coupling (red lines) the two resonances
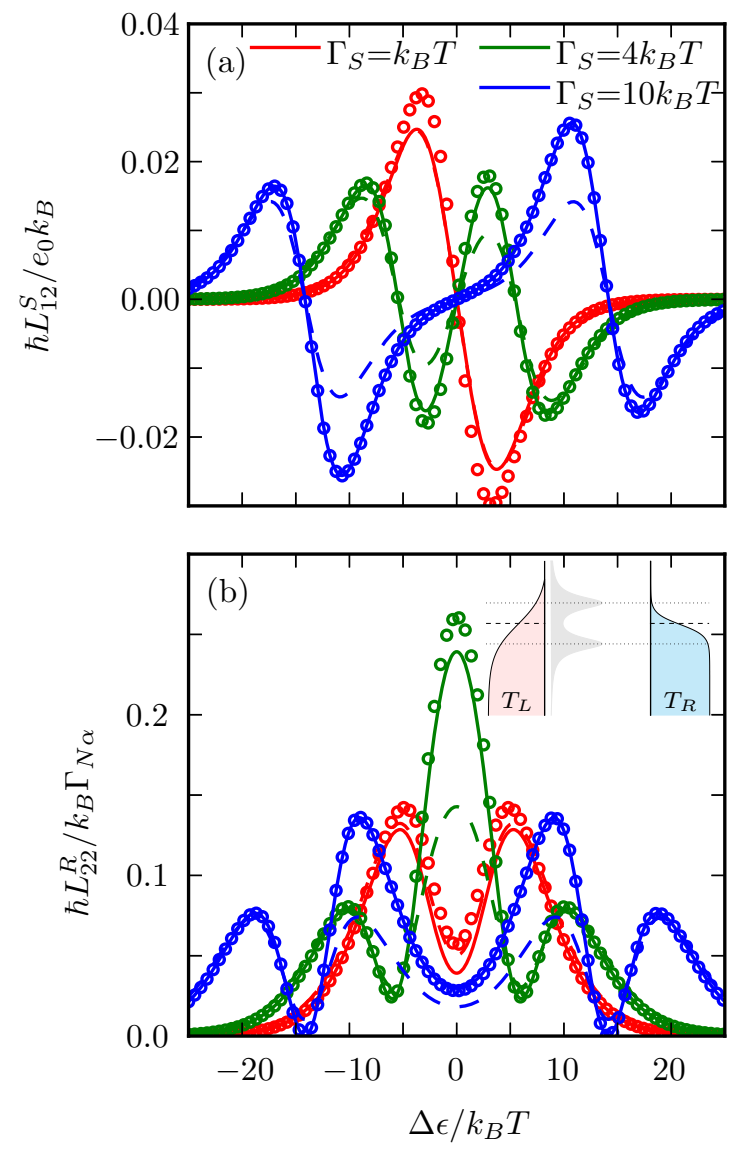

FIG. 4. (a) Linear current and (b) heat current coefficients $L_{12}^{S}$ and $L_{22}^{R}$ as a function of the detuning $\Delta \epsilon$ with the average temperature $T=5 \Gamma_{N \alpha} / k_{B}$ and all other parameters as in Fig. 2(a). The solid lines depict the transport coefficients obtained from the full master equation, the circles correspond to the reduced master equation, Eqs. (13) and (14), and the dashed lines correspond to the mapped model, see Eq. (10). The latter transport coefficients are scaled by a factor of $\alpha=0.37$, such that the effective linear current coefficient agrees with the one of the full model for $\Gamma_{S}=k_{B} T$. The inset sketches this mapped model of a single quantum dot with its on-site energies at the addition energies $\Delta E_{ \pm}$of the Cooper-pair splitter in the CAR regime.

effectively merge and the behavior resembles that of a single resonance with a minimum in the thermal conductance at zero detuning. The investigation (not shown) of the heat transport at the resonance $\Delta \epsilon=0$ demonstrates that the maximum is obtained for $\Gamma_{S}=4 k_{B} T$, so this quantity can be used as an indirect way to measure the strength of the nonlocal coupling.

A few comments on the origin of the deviation between the full result (solid lines) and the simplified model (dashed lines) are in order. In the simplified model, the peaks around the resonances of the thermoelectrical coefficient, see Fig. 4(a), are symmetric; this is not the case for the full results. The reason for the asymmetry is that the two resonances correspond to different Andreev levels which implies different energydependent weighting factors in front of the Lorentzians [92]. Similarly, for the thermal conductance, Fig. 4(b), the simplified model underestimates the height of the central peak. This can be again attributed to the fact that the central peak comes 
from the combined action of the two Andreev resonances and not from independent resonances as naively postulated in the simplified model. The differences between the two models are a specific signature of the nature of the Andreev bound states in the DQD system with respect to standard QD resonances.

In order to better elucidate this peculiar signature of the proximity in our system, we need to go beyond the simple two-resonance model. In particular, we can derive the current and the heat current for the case of symmetric detuning, $\epsilon_{L}=$ $-\epsilon_{R}$, in the reduced Hilbert space that is constituted by the empty state, the singly occupied states, and the singlet state as discussed around Eq. (9). Under this assumptions, we find explicit expressions for the charge and the heat current, as reported in the Appendix. From those equations, we can easily compute the nonlocal linear response coefficients following the definitions given in Eq. (7) and Eq. (8). One finds the linear response coefficients

$$
\frac{\hbar L_{12}^{S}}{e_{0} k_{B}}=-2 \frac{\Gamma_{N}}{k_{B} T} K\left(\Delta \tilde{\epsilon}, \Delta \tilde{\epsilon},-\sqrt{2} \tilde{\Gamma}_{S}\right)
$$

and

$$
\frac{\hbar L_{22}^{R}}{k_{B} \Gamma_{N}}=K\left(\Delta \tilde{\epsilon}^{2}+\frac{5 \tilde{\Gamma}_{S}^{2}}{2}, \Delta \tilde{\epsilon}^{2}+2 \tilde{\Gamma}_{S}^{2},-2 \sqrt{2} \tilde{\Gamma}_{S} \Delta \tilde{\epsilon}\right)
$$

where $\Delta \tilde{\epsilon}=\Delta \epsilon / 2 k_{B} T$ and $\tilde{\Gamma}_{S}=\Gamma_{S} / 2 k_{B} T$ are dimensionless parameters. Both quantities can be written in terms of the same universal function

$$
K(x, y, z)=\frac{x+y \cosh \tilde{\epsilon} \cosh \sqrt{2} \tilde{\Gamma}_{S}+z \sinh \tilde{\epsilon} \sinh \sqrt{2} \tilde{\Gamma}_{S}}{3\left(\cosh \tilde{\epsilon}+\cosh \sqrt{2} \tilde{\Gamma}_{S}\right)\left(2 \cosh \tilde{\epsilon}+\cosh \sqrt{2} \tilde{\Gamma}_{S}\right)} .
$$

Furthermore, in the reduced model the nonlocal linear transport coefficient $L_{21}^{R}$ fulfills the standard Onsager relation $L_{21}^{R}=T L_{12}^{S}$. This indirectly supports that the linear regime is associated with a sort of nonlocal reversibility condition. In Fig. 4, we show the transport coefficients of the reduced Hilbert space model with circles. In contrast to the mapped single-quantum-dot model (dashed lines), they capture the asymmetry in the peak heights of the linear current [panel (a)], and explain better the enhanced peaks of the linear heat current [panel (b)]. Moreover, the transport coefficients of the reduced model coincide with the ones of the full model (solid lines) in the case of a strong nonlocal coupling $\Gamma_{S} \gg k_{B} T$ (blue case) without any free parameter.

It is sometimes convenient to quantify the thermoelectricity in term of the Seebeck potential $\mu_{S}$. In the Appendix we compute the general formula for this quantity in Eq. (A4) in the reduced Hilbert space model, for symmetric detuning $\epsilon_{L}=-\epsilon_{R}$ and small temperature gradient $\delta T$. Taking the limit of $\Gamma_{S},|\Delta \epsilon| \gg k_{B} T$, one can further simplify the expression for the Seebeck potential to

$$
\mu_{S \pm} \approx\left(\Delta \epsilon \mp \sqrt{2} \Gamma_{S}\right) \frac{\delta T}{4 T}
$$

where the sign in $\mu_{S \pm}$ is simply determined by the sign of $\Delta \epsilon$. One can explain the above result for the Seebeck potential again in terms of the mapped quantum-dot model where the thermoelectric effect is determined by two different resonances located at $\Delta E_{ \pm}$. By definition, at the Seebeck potential, the thermocurrent generated by a temperature gradient vanishes. This is due to the fact that, at the addition energies $\Delta E_{ \pm}$, the electron distribution of the right normal lead (having the tendency to push electrons into the superconductor) is identical to the hole distribution on the left normal lead (having the tendency to pull electrons from the superconductor). Indeed the nonlocal thermocurrent is the result of these two competitive processes governed by the nonlocal Andreev bound state levels where the quasiparticle state on one dot is coupled with the quasihole state of the other, i.e., nonlocal particle-hole symmetry. This implies that the condition to calculate the Seebeck potential $\mu_{S}$, for a linear temperature gradient $\delta T$, is given by the two equations $f_{R}\left(\Delta E_{ \pm}\right)=1-f_{L}\left(-\Delta E_{\mp}\right)$, where the Fermi functions are computed at the equilibrium temperature $T=\left(T_{R}+T_{L}\right) / 2$. The solution of these two conditions returns exactly the two results of Eq. (15), providing a physical interpretation for the full formula given in the Appendix.

\section{B. Nonlocal cooling}

We conclude this section with a final remark on the possibility to obtain a cooling cycle. When a thermoelectrical device is operated near the reversibility condition, the thermoelectrical cycle can be inverted in order to get a cooling cycle [74]. Inspecting the level structure sketched in Fig. 3(b) one would expect nonlocal cooling at finite chemical potential. With cooling of a normal lead, we mean that electrons are either added below its Fermi level or extracted above its Fermi level. In particular, we expect that in the linear regime, by slightly moving the chemical potential around the values $\mu_{S \pm}$, the nonlocal thermogenerator could turn into a nonlocal cooler and our thermoelectrical engine becomes a cooling device (Peltier cooling).

In order to verify this mechanism, we first give a closer look to the heat current $\dot{Q}_{R}$ of Eq. (A1), linearizing the chemical potential $\mu \equiv \mu_{S}+\delta \mu_{S}$ around the nonlocal Seebeck potential $\mu_{S}$ of Eq. (A4). For simplicity, we consider equal temperatures, $T=T_{L}=T_{R}$, a condition for which nonlocal cooling is still possible. One finds that $\left.\dot{Q}_{R}\right|_{\delta T=0} \approx L_{21}^{R} \delta \mu_{S} / e_{0}$, which shows clearly that the heat flux changes its sign with the sign of $\delta \mu_{S}$. So, when the chemical potential crosses the nonlocal Seebeck potential, indeed, the system reverses the heat flux. In the presence of a small temperature gradient between the normal leads this heat-flux reversal corresponds to the conversion of a thermoelectrical generator into a Peltier cooler.

This scenario is further supported by the behavior of the total electric power $P \equiv-\mu I_{S} / e_{0}$ generated around the nonlocal Seebeck potential $\mu_{S}$. Indeed, by linearizing in $\delta \mu_{S}$ the thermopower becomes $P \approx L_{12}^{S} \delta T \delta \mu_{S} / e_{0}$ where $\delta T$ is the temperature difference. Coherently, one sees that the crossing of the nonlocal Seebeck potential, $\delta \mu_{S} \rightarrow-\delta \mu_{S}$, results in a sign change of the total power and, thus, entails a change between power generation to dissipation, as expected from consistency with general thermodynamical arguments for the scenario described so far.

We will discuss the general behavior of nonlocal thermoelectricity in more depth in Sec. IV, which treats the nonlinear regime, and address, therein, quantitatively the thermodynamical performances of the thermopower and the cooling. Furthermore, we will see that nonlocal cooling, indeed, sets 

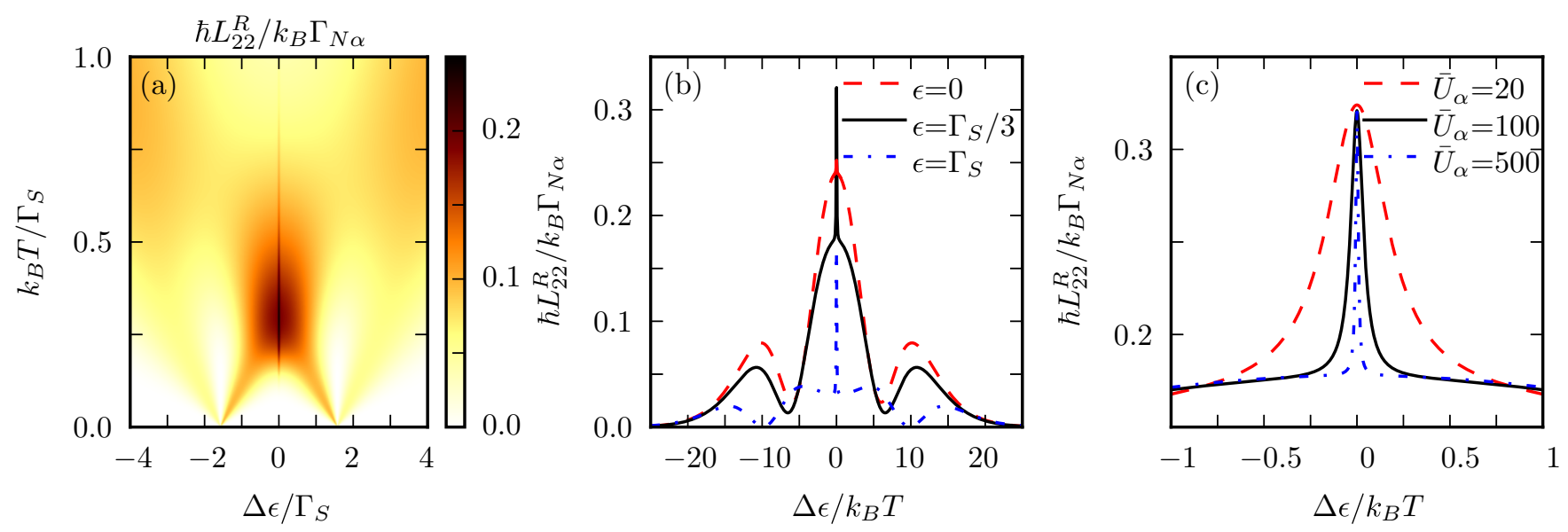

FIG. 5. (a) Linear heat current coefficient $L_{22}^{R}$ (which for $\delta V=0$ is proportional to the heat current $\dot{Q}_{R}$ ) as a function of the detuning $\Delta \epsilon$ and the average temperature $T$ for finite intradot Coulomb energy $U_{\alpha}=100 \Gamma_{S \alpha}, \Gamma_{S}=\Gamma_{S \alpha} / 3=4 k_{B} T$, and $k_{B} T=10 \Gamma_{N \alpha}$. Here, the level energies $\epsilon_{L}=\epsilon-\Delta \epsilon / 2$ and $\epsilon_{R}=\epsilon+\Delta \epsilon / 2$ are centered around the average value $\epsilon=\Gamma_{S} / 3$. (b) Dependence on the average level energies $\epsilon$ and (c) the interdot couplings $\bar{U}_{\alpha} \equiv U_{\alpha} / \Gamma_{S \alpha}$.

in at $\mu \approx \mu_{S \pm}$ also well beyond the discussed linear regime. However before doing so, we discuss in the following section the effect of finite Coulomb interaction on the heat transport properties at the nonlocal resonance.

\section{Effect of finite Coulomb interaction}

Thus far, we have restricted our analysis to the case of infinite local Coulomb interaction in the QDs, so that the double occupation of the individual dots is forbidden. Relaxing this condition and considering finite values for $U_{\alpha}$ opens up the possibility of a local exchange of Cooper pairs between the superconductor and the system (both electrons in the Copper pair tunnel to/from the same dot) [93]. This includes the possibility to consider different virtual transitions involving a nonlocal resonance. In this way, electrons can transfer energy between the normal leads via elastic cotunneling; in the subgap regime $(|\Delta| \rightarrow \infty)$ elastic cotunneling is not mediated by quasiparticles with energies larger then $\Delta$, but rather by multiple exchanges of local and nonlocal Cooper pairs triggered by $\Gamma_{S}$ and $\Gamma_{S \alpha}$. Thus, the finite intradot Coulomb interaction can increase the heat current $\dot{Q}_{R}$, while the current $I_{S}$ through the superconductor remains unaffected. In particular, an electron with spin $\sigma$ above the chemical potential of the left normal lead may tunnel with the rate $\Gamma_{N L}$ into the left dot and occupy the state $|L \sigma\rangle$. Then a Cooper pair may split nonlocally with the coupling $\Gamma_{S}$ into the triply occupied state $|t R \sigma\rangle=$ $d_{R \sigma}^{\dagger} d_{L \uparrow}^{\dagger} d_{L \downarrow}^{\dagger}|0\rangle$ followed by a local Cooper-pair recombination with the rate $\Gamma_{S L}$. Finally, the electron leaves the dot with the rate $\Gamma_{N R}$ via the right normal lead, heating up the right lead. The process can also proceed differently with the local coupling operating before the nonlocal one. In this process the electron is effectively transferred to the state $|R \sigma\rangle$ of the right dot with no net current in the superconductor. This shows again that, due to nonlocality, the resonant behavior of the heat does not necessarily affect the charge current. The aforementioned mechanism can be identified in the thermal transport at finite interaction such as in Fig. 5(a), where the thermal transport coefficient $L_{22}^{R}$ is shown as a function of the detuning $\Delta \epsilon$ and the average temperature $T$. The thermal conductance $L_{22}^{R}$ describes how the heat current flows between the two normal terminals for $\mu=0$. A remarkable feature is the narrow resonance at $\Delta \epsilon=0$. This resonance is absent for infinite local Coulomb interactions and its linewidth increases with the scaling $\Gamma_{\alpha} / U_{\alpha}$, indicating its origin in the elastic cotunneling mechanism.

In Fig. 5(c) one can appreciate that for increasing intradot Coulomb interaction, the cotunneling peak becomes narrower, while its height remains unaffected. For these calculations the average quantum dot level has been chosen to be $\epsilon \equiv$ $\left(\epsilon_{L}+\epsilon_{R}\right) / 2=\Gamma_{S} / 3$ since for $\epsilon=0$ the resonance is less pronounced [panel (b)].

\section{NONLOCAL THERMOELECTRIC POWER}

So far, we have studied the transport coefficients for equal chemical potentials $\mu_{R}=\mu_{L}=\mu_{S C}=0$. In this case there is no power generation. For any circuital element, electrical work is performed if the charge carriers gain potential energy by flowing against an increasing chemical potential. Therefore, while keeping the chemical potential of the superconductor at zero, for reference, we now consider the normal leads at nonzero values of $\mu$. The corresponding generated work or thermopower $P \equiv-\mu I_{S} / e_{0}$ reflects the potential energy that an electron gains [94]. Upon increasing $\mu$ from a finite value, which still allows such counterflow, to even larger ones, the flow of electrons will come at some specific value to a standstill. Increasing $\mu$ further will change the sign of the current. Then, the thermoelectric element becomes dissipative and the electrons flow in the direction of the potential drop. In the inverted regime, a cooling effect can be also found before, at even higher voltages, where the fully dissipative regime dominates.

Figure 6(a) shows the nonlocal thermopower as a function of the chemical potential and the detuning. As expected, the current becomes dissipative (negative power) for sufficiently large absolute values of $\mu$. Nevertheless, there exist regions, namely the triangular ones in red, in which the Cooper-pair splitter effectively generates positive thermopower and acts 

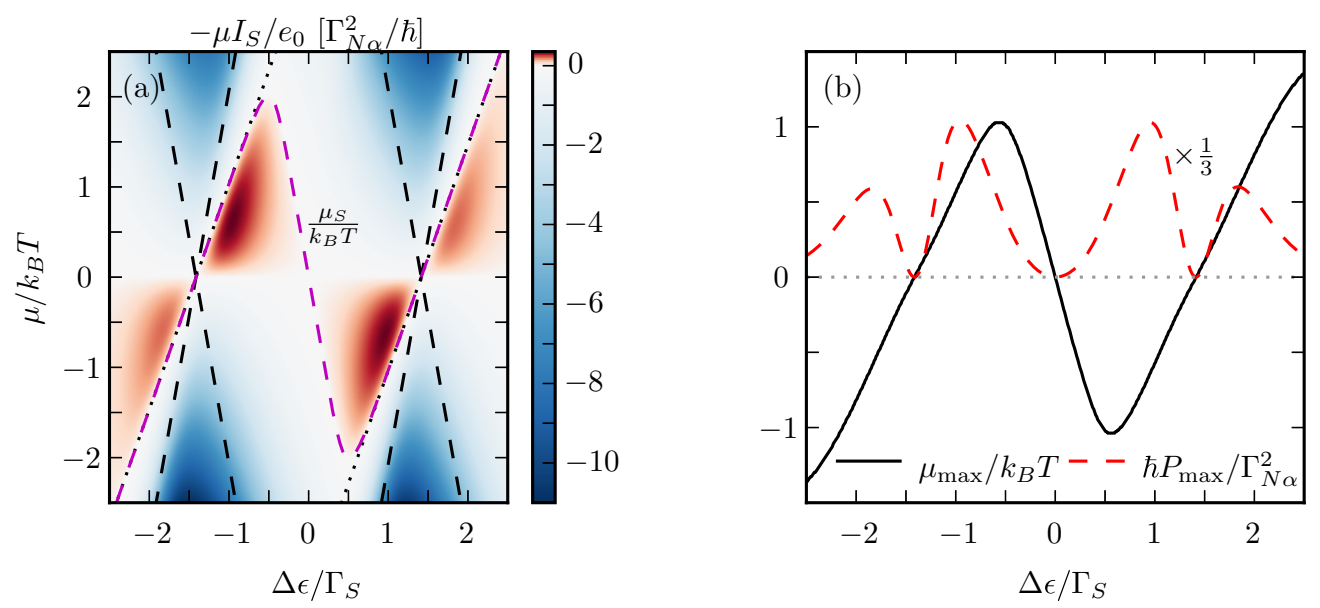

FIG. 6. (a) Thermopower $P=-\mu I_{S} / e_{0}$ as a function of the detuning $\Delta \epsilon \equiv \epsilon_{R}-\epsilon_{L}$ and the chemical potential $\mu \equiv \mu_{L}=\mu_{R}$, choosing the average level energy to be zero, i.e., $\epsilon_{R}+\epsilon_{L}=0$, and $k_{B} T_{L}=3 k_{B} T_{R}=15 \Gamma_{N \alpha}$. Other parameters are chosen as in Fig. 2 (a). The dashed black lines indicate where the addition energies $\Delta E_{ \pm}$are in resonance with the chemical potential $\mu$, while the dotted lines correspond to the estimated nonlocal Seebeck potential $\mu_{S \pm}$ given in Eq. (15). The dashed purple line corresponds to the condition (A3) for the nonlocal Seebeck potential $\mu_{S}$. (b) Maximal power $P_{\max }=\max _{\mu} P(\Delta \epsilon, \mu)$ (dashed line) and chemical potential $\mu_{\max }$ corresponding to the maximal power (solid line) for fixed detuning.

as a thermogenerator. Furthermore, one can appreciate in this figure that the thermogenerator regime (red zones) is delimited by the nonlocal Seebeck formula Eq. (A3) (dashed purple line) derived in Appendix, which can be approximated around the Andreev resonances by Eq. (15) (black dotted line).

In Fig. 6(b), we show the maximum generated power $P_{\max }$ (red dashed line) and the corresponding nonlocal Seebeck potential $\mu_{\max }$ (solid line) for which this maximum is obtained. The maximum generated power is relatively small, $P_{\max } \approx \Gamma_{N \alpha}^{2} / 3 \hbar$, and decaying for large detuning.

Finally we discuss the thermoelectric efficiencies for power generation and cooling. For the nonlocal power generation, the efficiency $r_{\text {eng }}=\left(P /\left|\dot{Q}_{L}\right|\right) / \eta_{C}$ is given by the power $P>0$ generated in the system per extracted heat flow $-\dot{Q}_{L}>0$ from the warmer normal lead [58]. It is normalized to the Carnot efficiency of a heat engine $\eta_{C}=1-T_{R} / T_{L}$, which is bounded between 0 and 1. Similarly, the cooling power $r_{\text {fri }}=\left(\dot{Q}_{R} / P\right) / \eta_{\text {fri }}$ is defined as the heat flow $\dot{Q}_{R}<0$ extracted from the cold reservoir per absorbed power. We compare it with the ideal efficiency of a refrigerator $\eta_{\mathrm{fri}}=T_{R} /\left(T_{L}-T_{R}\right)$, which can be larger than one. The combined efficiency

$$
r_{\text {therm }}= \begin{cases}r_{\text {eng }}, & P>0, \\ -r_{\text {fri }}, & \dot{Q}_{R}<0,\end{cases}
$$

is depicted in Fig. 7 as a function of the detuning and the chemical potential, where positive values (red) correspond to power generation and negative values (blue) to cooling of the right normal lead. Close to the lines where the thermoelectric current vanishes, $\mu \propto(\delta T / 4 T) \Delta \epsilon$, see Eq. (15), the system has a very high efficiency and represents an almost reversible thermoelectric generator (red shaded area). However, the power generated under this condition is rather small. Therefore, as usual, there is a trade-off between high efficiency and high output. In particular at the nonlocal Seebeck potential, where the thermoelectric current and the thermopower vanish, one generically expects that the thermoelectrical machine becomes reversible and reaches the Carnot efficiency.

The fact that the thermal machine operates nearly at the Carnot efficiency for some finite $\mu \neq 0$ suggests that the system can become a cooling device. This happens, indeed, in the blue shaded region bounded by the nonlocal Andreev resonance at $\mu \propto \Delta \epsilon / 2$, where the colder lead is further cooled due to nonlocal Cooper-pair tunneling.

\section{CONCLUSIONS}

Nonlocal thermoelectric effects in a double-dot Cooperpair splitter have been investigated. Thermoelectricity properties are determined by the nonlocal breaking of the particle-hole symmetry which is realized in the hybrid threeterminal structure in the presence of a temperature gradient.

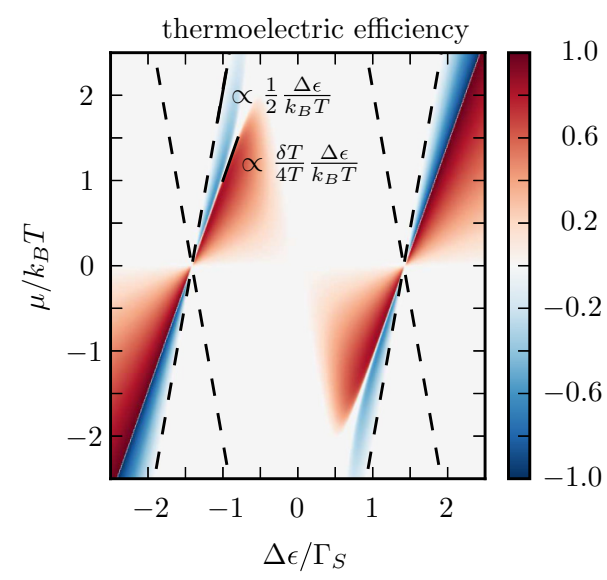

FIG. 7. Power generation efficiency $P /\left(-\dot{Q}_{L}\right)$ (red) for $P>0$, and cooling efficiency $\dot{Q}_{R} / P$ (blue) for $\dot{Q}_{R}<0$ normalized by the corresponding Carnot efficiencies; see Eq. (16). In order to depict both efficiencies in the same plot, the latter is multiplied by a factor of -1 . All parameters are as in Fig. 6. 
Intriguingly, we demonstrated that the superconductor can mediate coherent heat transfer between the normal leads. The rich phenomenology can be easily interpreted in terms of a simple model consisting of two resonances located at the nonlocal Andreev bound state addition energies. However, this model has some limitations and the full model is required to get accurate results for the thermal transport. In particular, the Andreev nature of the resonances is reflected in a different energy dependence of those resonances. In comparison to the simple model, we predict an enhancement of the heat transferred between the normal lead at resonance for $\Gamma_{S}=$ $4 k_{B} T$. Finally, at the nonlocal resonance for finite Coulomb interaction an extra resonance is located in the heat transport as a consequence of virtual transitions to triple occupied states. When applying a load between the normal leads and the superconducting one, the Cooper-pair splitter can perform work and convert heat current into electric current with nearly Carnot efficiency. The detuning can be used as control knob to turn the nonlocal power generator to a heat pump and cool the colder normal lead via nonlocal Cooper-pair tunneling.

Note added. Recently, we became aware of a related work by Sánchez et al. [95] and also works by Kirsanov et al. [96] and Pershoguba et al. [97].

\section{ACKNOWLEDGMENTS}

R.H. acknowledges financial support from the Carl-ZeissStiftung. This work was supported by the Spanish Ministry of Economy and Competitiveness via Grants No. MAT201786717-P and No. MAT2016-82015-REDT. W.B. acknowledges financial support from the DFG through SPP 1538 "Spin Caloric Transport." A.B. and F.G. acknowledge the European Research Council under the European Union's Seventh Framework Program (FP7/2007-2013)/ERC Grant Agreement No. 615187-COMANCHE and the Tuscany Region under the PAR FAS 2007-2013, FAR-FAS 2014 call, project SCIADRO for partial financial support. A.B. acknowledges the CNR-CONICET cooperation program "Energy conversion in quantum nanoscale hybrid devices" and the Royal Society through the International Exchanges between the UK and Italy (Grant No. IES R3 170054). M.G. acknowledges the hospitality of Scuola Normale Superiore, Pisa.

\section{APPENDIX: CURRENT AND HEAT CURRENT FOR STRONG NONLOCAL COUPLING}

In this Appendix, we provide analytical expressions for the current, Eq. (5), and the heat current, Eq. (6), assuming symmetric detuning, $\epsilon_{L}=-\epsilon_{R}$, and equal couplings to the normal leads, $\Gamma_{N} \equiv \Gamma_{N \alpha}$. As discussed in the main text, we derive these quantities in the reduced Hilbert space spanned by the empty state, the singly occupied states, and the singlet state which is a good approximation at nonlocal resonance and for strong intradot Coulomb interactions $U_{R}, U_{L} \rightarrow \infty$. By using the general method presented in Sec. II, we find the expressions

$$
\begin{aligned}
I_{R} & =\frac{e_{0}}{\hbar} \frac{N_{I}}{D}, \quad I_{S}=-2 I_{R} \\
\dot{Q}_{R} & =-\frac{1}{\hbar} \frac{N_{E}}{D}+\frac{1}{e_{0}}\left(\frac{\Delta \epsilon}{2}-\mu\right) I_{R}
\end{aligned}
$$

with the numerator

$$
N_{I}=\Gamma_{N}\left(\sum_{p, q} g_{p}^{p, q}-2\right)\left[\left(\bar{g}_{+}^{+,+}-g_{-}^{-,-}\right)\left(\bar{g}_{+}^{+,-}-g_{-}^{-,+}\right)-9\right]
$$

of the current and the numerator of the energy current

$$
\begin{aligned}
N_{E}= & \frac{9 \Gamma_{N} \Gamma_{S}}{2 \sqrt{2}} \sum_{p} p\left[g_{-}^{-,-p} g_{+}^{+, p}+\left(2+g_{-}^{-, p} g_{+}^{+,-p}\right)\right. \\
& \left.\times\left(g_{-}^{-,-p}+g_{+}^{+, p}\right)\right] .
\end{aligned}
$$

The common denominator is given by

$$
\begin{aligned}
D= & 32+2 \sum_{p, q} g_{p}^{p, q}\left[3+g_{p}^{p, q}\left(g_{p}^{p,-q}-\bar{g}_{-p}^{-p, q}\right)\right] \\
& +\sum_{p}\left[10 g_{p}^{p,-} g_{p}^{p,+}+g_{-}^{-, p}\left(5 g_{+}^{+,-p}-17 g_{+}^{+, p}\right)\right. \\
& \left.-5 g_{p}^{p, p} g_{p}^{p,-p}\left(g_{-p}^{-p,-p}+g_{-p}^{-p, p}\right)\right] .
\end{aligned}
$$

Here, $g_{\alpha}^{p, q}=\left\{1+\exp \left[\left(p \frac{\Delta \epsilon}{2}+q \frac{\Gamma_{S}}{\sqrt{2}}-\mu\right) / k_{B} T_{\alpha}\right]\right\}^{-1}$ compactly denotes the Fermi function evaluated at the Andreev bound state addition energies with $p, q \in\{-1,1\}$, and $\bar{g}_{\alpha}^{p, q} \equiv 1-$ $g_{\alpha}^{p, q}$. For the sake of a compact notation, we also identified the terminals $\alpha=L, R$ with the values $\alpha=\mp$. The corresponding current and heat current on the left normal lead follow from the mutual replacement $\left\{\epsilon_{L} \leftrightarrow \epsilon_{R}, T_{L} \leftrightarrow T_{R}\right\}$. We note that $N_{I}$ and $D$ are unaffected under this transformation since the currents through the normal leads are identical, $I_{L}=I_{R}$. On the contrary, $N_{E}$ experiences a change in sign leading to the energy conservation $\dot{Q}_{L}+\dot{Q}_{R}=\mu I_{S}$.

A closer inspection of the numerator $N_{I}$ reveals that the current through the superconductor only vanishes if the term in its first parenthesis nullifies, since the denominator is always finite. This gives us the condition

$$
2=\left.\sum_{p, q} g_{p}^{p, q}\right|_{\mu=\mu_{S}}
$$

which defines implicitly the nonlocal Seebeck potential $\mu_{S}$. Figure 6 of the main text visualizes that this expression $\mu_{S}$ (dashed purple line) asymptotically approaches the estimates $\mu_{S \pm}$ (dotted black lines), Eq. (15), of the mapped single-quantum-dot model. This asymptotic behavior, approximated as

$$
\mu_{S} \approx\left[\Delta \epsilon-\frac{\sqrt{2} \Gamma_{S} \sinh \left(\frac{\Delta \epsilon}{2 k_{B} T}\right) \sinh \left(\frac{\Gamma_{S}}{\sqrt{2} k_{B} T}\right)}{1+\cosh \left(\frac{\Delta \epsilon}{2 k_{B} T}\right) \cosh \left(\frac{\Gamma_{S}}{\sqrt{2} k_{B} T}\right)}\right] \frac{\delta T}{4 T},
$$

follows from the linearization of Eq. (A5) in $\mu_{S}$ and the temperature difference $\delta T$. In the limit $\Gamma_{S},|\Delta \epsilon| \gg k_{B} T$, this reduces for $\operatorname{sgn}(\Delta \epsilon)= \pm 1$ to the branches $\mu_{S \pm}$, respectively. 
[1] G. B. Lesovik, T. Martin, and G. Blatter, Eur. Phys. J. B 24, 287 (2001).

[2] S. Russo, M. Kroug, T. M. Klapwijk, and A. F. Morpurgo, Phys. Rev. Lett. 95, 027002 (2005).

[3] A. Martín-Rodero and A. L. Yeyati, Adv. Phys. 60, 899 (2011).

[4] S. Roddaro, A. Pescaglini, D. Ercolani, L. Sorba, F. Giazotto, and F. Beltram, Nano Res. 4, 259 (2011).

[5] F. Giazotto, P. Spathis, S. Roddaro, S. Biswas, F. Taddei, M. Governale, and L. Sorba, Nat. Phys. 7, 857 (2011).

[6] J. Schindele, A. Baumgartner, and C. Schönenberger, Phys. Rev. Lett. 109, 157002 (2012).

[7] L. Romeo, S. Roddaro, A. Pitanti, D. Ercolani, L. Sorba, and F. Beltram, Nano Lett. 12, 4490 (2012).

[8] A. Das, Y. Ronen, M. Heiblum, D. Mahalu, A. V. Kretinin, and H. Shtrikman, Nat. Commun. 3, 1165 (2012).

[9] B. Braunecker, P. Burset, and A. Levy Yeyati, Phys. Rev. Lett. 111, 136806 (2013).

[10] F. Rossella, A. Bertoni, D. Ercolani, M. Rontani, L. Sorba, F. Beltram, and S. Roddaro, Nat. Nanotechnol. 9, 997 (2014).

[11] M.-S. Choi, Phys. Rev. B 89, 045137 (2014).

[12] K. Sato and Y. Tserkovnyak, Phys. Rev. B 90, 045419 (2014).

[13] R. S. Deacon, A. Oiwa, J. Sailer, S. Baba, Y. Kanai, K. Shibata, K. Hirakawa, and S. Tarucha, Nat. Commun. 6, 7446 (2015).

[14] P. Marra, R. Citro, and A. Braggio, Phys. Rev. B 93, 220507 (2016).

[15] J. Tiira, E. Strambini, M. Amado, S. Roddaro, P. San-Jose, R. Aguado, F. S. Bergeret, D. Ercolani, L. Sorba, and F. Giazotto, Nat. Commun. 8, 14984 (2017).

[16] G. Blasi, F. Taddei, V. Giovannetti, and A. Braggio, arXiv:1808.09709.

[17] J. Linder and J. W. A. Robinson, Nat. Phys. 11, 307 (2015).

[18] C. Monroe, Nature (London) 416, 238 (2002).

[19] T. D. Ladd, F. Jelezko, R. Laflamme, Y. Nakamura, C. Monroe, and J. L. O'Brien, Nature (London) 464, 45 (2010).

[20] D. Beckmann, H. B. Weber, and H. v. Löhneysen, Phys. Rev. Lett. 93, 197003 (2004).

[21] L. Hofstetter, A. Geresdi, M. Aagesen, J. Nygård, C. Schönenberger, and S. Csonka, Phys. Rev. Lett. 104, 246804 (2010).

[22] P. Trocha and I. Weymann, Phys. Rev. B 91, 235424 (2015).

[23] K. Wrześniewski, P. Trocha, and I. Weymann, J. Phys.: Condens. Matter 29, 195302 (2017).

[24] K. Bocian, W. Rudziński, and I. Weymann, Phys. Rev. B 97, 195441 (2018).

[25] M.-S. Choi, C. Bruder, and D. Loss, Phys. Rev. B 62, 13569 (2000).

[26] P. Recher, E. V. Sukhorukov, and D. Loss, Phys. Rev. B 63, 165314 (2001).

[27] O. Sauret, D. Feinberg, and T. Martin, Phys. Rev. B 70, 245313 (2004)

[28] L. Hofstetter, S. Csonka, J. Nygard, and C. Schönenberger, Nature (London) 461, 960 (2009).

[29] L. G. Herrmann, F. Portier, P. Roche, A. L. Yeyati, T. Kontos, and C. Strunk, Phys. Rev. Lett. 104, 026801 (2010).

[30] J. Schindele, A. Baumgartner, R. Maurand, M. Weiss, and C. Schönenberger, Phys. Rev. B 89, 045422 (2014).

[31] G. Fülöp, F. Domínguez, S. d'Hollosy, A. Baumgartner, P. Makk, M. H. Madsen, V. A. Guzenko, J. Nygård, C. Schönenberger, A. Levy Yeyati, and S. Csonka, Phys. Rev. Lett. 115, 227003 (2015).
[32] B. Probst, F. Domínguez, A. Schroer, A. L. Yeyati, and P. Recher, Phys. Rev. B 94, 155445 (2016).

[33] R. Hussein, L. Jaurigue, M. Governale, and A. Braggio, Phys. Rev. B 94, 235134 (2016).

[34] R. Hussein, A. Braggio, and M. Governale, Phys. Status Solidi B 254, 1600603 (2017).

[35] H. B. Radousky and H. Liang, Nanotechnology 23, 502001 (2012).

[36] B. Roche, P. Roulleau, T. Jullien, Y. Jompol, I. Farrer, D. Ritchie, and D. Glattli, Nat. Commun. 6, 6738 (2015).

[37] B. Sothmann, R. Sánchez, and A. N. Jordan, Nanotechnology 26, 032001 (2015).

[38] H. Thierschmann, R. Sánchez, B. Sothmann, F. Arnold, C. Heyn, W. Hansen, H. Buhmann, and L. W. Molenkamp, Nat. Nanotechnol. 10, 854 (2015).

[39] J. Mastomäki, S. Roddaro, M. Rocci, V. Zannier, D. Ercolani, L. Sorba, I. J. Maasilta, N. Ligato, A. Fornieri, E. Strambini, and F. Giazotto, Nano Res. 10, 3468 (2017).

[40] P. Virtanen and T. T. Heikkilä, Phys. Rev. Lett. 92, 177004 (2004).

[41] P. Machon, M. Eschrig, and W. Belzig, Phys. Rev. Lett. 110, 047002 (2013).

[42] P. Machon, M. Eschrig, and W. Belzig, New J. Phys. 16, 073002 (2014).

[43] A. Ozaeta, P. Virtanen, F. S. Bergeret, and T. T. Heikkilä, Phys. Rev. Lett. 112, 057001 (2014).

[44] F. Giazotto, P. Solinas, A. Braggio, and F. S. Bergeret, Phys Rev. Appl. 4, 044016 (2015).

[45] F. Giazotto, T. T. Heikkilä, and F. S. Bergeret, Phys. Rev. Lett. 114, 067001 (2015).

[46] J. Linder and M. E. Bathen, Phys. Rev. B 93, 224509 (2016).

[47] K. I. Wysokiński, J. Phys.: Condens. Matter 24, 335303 (2012).

[48] S.-Y. Hwang, R. López, and D. Sánchez, Phys. Rev. B 91, 104518 (2015).

[49] S.-Y. Hwang, R. López, and D. Sánchez, Phys. Rev. B 94, 054506 (2016).

[50] P. Trocha and J. Barnaś, Phys. Rev. B 95, 165439 (2017).

[51] C. Van den Broeck, Phys. Rev. Lett. 95, 190602 (2005).

[52] B. Muralidharan and M. Grifoni, Phys. Rev. B 85, 155423 (2012).

[53] N. Brunner, N. Linden, S. Popescu, and P. Skrzypczyk, Phys. Rev. E 85, 051117 (2012).

[54] L. A. Correa, J. P. Palao, G. Adesso, and D. Alonso, Phys. Rev. E 87, 042131 (2013).

[55] F. Mazza, R. Bosisio, G. Benenti, V. Giovannetti, R. Fazio, and F. Taddei, New J. Phys. 16, 085001 (2014).

[56] R. S. Whitney, Phys. Rev. Lett. 112, 130601 (2014).

[57] P. P. Hofer, J.-R. Souquet, and A. A. Clerk, Phys. Rev. B 93, 041418 (2016).

[58] G. Benenti, G. Casati, K. Saito, and R. S. Whitney, Phys. Rep. 694, 1 (2017).

[59] S. Seah, S. Nimmrichter, and V. Scarani, Phys. Rev. E 98, 012131 (2018).

[60] L. Karwacki and P. Trocha, Phys. Rev. B 94, 085418 (2016).

[61] P. A. Erdman, F. Mazza, R. Bosisio, G. Benenti, R. Fazio, and F. Taddei, Phys. Rev. B 95, 245432 (2017).

[62] Z. Cao, T.-F. Fang, L. Li, and H.-G. Luo, Appl. Phys. Lett. 107, 212601 (2015).

[63] A. V. Rozhkov and D. P. Arovas, Phys. Rev. B 62, 6687 (2000). 
[64] T. Meng, S. Florens, and P. Simon, Phys. Rev. B 79, 224521 (2009).

[65] J. Eldridge, M. G. Pala, M. Governale, and J. König, Phys. Rev. B 82, 184507 (2010).

[66] A. Braggio, M. Governale, M. G. Pala, and J. König, Solid State Commun. 151, 155 (2011).

[67] B. Sothmann, S. Weiss, M. Governale, and J. König, Phys. Rev. B 90, 220501 (2014).

[68] S. Weiss and J. König, Phys. Rev. B 96, 064529 (2017).

[69] N. Walldorf, C. Padurariu, A.-P. Jauho, and C. Flindt, Phys. Rev. Lett. 120, 087701 (2018).

[70] F. J. Kaiser, M. Strass, S. Kohler, and P. Hänggi, Chem. Phys. 322, 193 (2006).

[71] D. Darau, G. Begemann, A. Donarini, and M. Grifoni, Phys. Rev. B 79, 235404 (2009).

[72] P. P. Hofer, M. Perarnau-Llobet, L. D. M. Miranda, G. Haack, R. Silva, J. B. Brask, and N. Brunner, New J. Phys. 19, 123037 (2017).

[73] J. O. González, L. A. Correa, G. Nocerino, J. P. Palao, D. Alonso, and G. Adesso, Open Syst. Inf. Dyn. 24, 1740010 (2017).

[74] F. Mazza, S. Valentini, R. Bosisio, G. Benenti, V. Giovannetti, R. Fazio, and F. Taddei, Phys. Rev. B 91, 245435 (2015).

[75] Notice that at the nonlocal resonance even for a finite voltage bias between the normal leads essentially no current will flow between them in the limit of strong intradot Coulomb interaction.

[76] K. Saito and A. Dhar, Phys. Rev. Lett. 99, 180601 (2007).

[77] K. Saito and Y. Utsumi, Phys. Rev. B 78, 115429 (2008).

[78] R. Hussein and S. Kohler, Phys. Rev. B 89, 205424 (2014).

[79] D. A. Bagrets and Yu. V. Nazarov, Phys. Rev. B 67, 085316 (2003).
[80] A. Braggio, J. König, and R. Fazio, Phys. Rev. Lett. 96, 026805 (2006).

[81] C. Flindt, T. Novotný, A. Braggio, M. Sassetti, and A.-P. Jauho, Phys. Rev. Lett. 100, 150601 (2008).

[82] C. Flindt, T. Novotný, A. Braggio, and A.-P. Jauho, Phys. Rev. B 82, 155407 (2010).

[83] R. Sánchez and M. Büttiker, Europhys. Lett. 100, 47008 (2012).

[84] S. Gasparinetti, P. Solinas, A. Braggio, and M. Sassetti, New J. Phys. 16, 115001 (2014).

[85] In principle a full investigation at finite $U_{\alpha}$ can be performed; see Ref. [33].

[86] The simplicity of this picture is due to the absence of the double occupancy of the individual dots, which is energetically forbidden by the strong interaction.

[87] C. W. J. Beenakker, Phys. Rev. B 44, 1646 (1991).

[88] M. Turek and K. A. Matveev, Phys. Rev. B 65, 115332 (2002).

[89] N. Nakpathomkun, H. Q. Xu, and H. Linke, Phys. Rev. B 82, 235428 (2010).

[90] Y. Dubi and M. Di Ventra, Rev. Mod. Phys. 83, 131 (2011).

[91] C. Eltschka, H. Thierschmann, H. Buhmann, and J. Siewert, Phys. Status Solidi A 213, 626 (2016).

[92] J. Splettstoesser, M. Governale, J. König, F. Taddei, and R. Fazio, Phys. Rev. B 75, 235302 (2007).

[93] In order to have a finite nonlocal coupling $\Gamma_{S}$ both the two local couplings $\Gamma_{S \alpha}$ with the superconductor need to be finite as $\Gamma_{S} \propto$ $\sqrt{\Gamma_{S R} \Gamma_{S L}}$.

[94] M. Rey and F. Sols, Phys. Rev. B 70, 125315 (2004).

[95] R. Sánchez, P. Burset, and A. L. Yeyati, Phys. Rev. B 98, 241414 (2018).

[96] N. S. Kirsanov, Z. B. Tan, D. S. Golubev, P. J. Hakonen, and G. Lesovik, arXiv:1806.09838.

[97] S. S. Pershoguba and L. I. Glazman, arXiv:1901.10065. 